\title{
Spin-Relaxation Effects on the EPR Spectrum of Gaseous Hydrogen Atoms
}

\author{
Robert L. Brown \\ Institute for Materials Research, National Bureau of Standards, Washington, D.C. 20234
}

(November 19, 1971)

\begin{abstract}
A theoretical analysis is presented of the relaxation processes which lead to the magnetization of gaseous hydrogen atoms in a strong magnetic field. Experiments are described which demonstrate that the spin-lattice relaxation time in a pure $\mathrm{H}_{2}$ carrier can be of the order of $10 \mathrm{~ms}$. From the effects of traces of $\mathrm{O}_{2}$ on the hydrogen atom EPR spectrum, a $\mathrm{H}-\mathrm{O}_{2}$ spin-exchange collision cross section of $(8.0 \pm 2.5) \times 10^{-16} \mathrm{~cm}^{2}$ was derived.
\end{abstract}

Key words: Electron spin resonance; hydrogen atoms; spin-exchange; spin-relaxation.

\section{Introduction}

It has been demonstrated that gaseous nitrogen atoms can have a spin-lattice relaxation time $T_{1}$ of the order of several milliseconds [1]. ${ }^{1}$ In high speed discharge-flow systems with EPR atom detection, this $T_{1}$ can be longer than the transit time of the atoms between the discharge and the EPR microwave cavity. Anomalously small EPR ałsorption intensities will arise in this situation because the atoms entering the cavity will not be completely magnetized. Decreasing the gas flow rate or decreasing $T_{1}$ by adding a paramagnetic impurity like $\mathrm{O}_{2}$ will increase the magnetization at the cavity. The EPR signal will thus increase even if the atom concentration in the cavity remains constant. To measure atom concentrations quantitatively by EPR under such conditions one must know how far the magnetization lies from equilibrium or else produce an equilibrium condition by adding sufficient paramagnetic impurity.

In general, large values of $T_{1}$ are to be expected for $S$-state atoms because of the weak coupling of the electron spin to the rest of the atom and hence, through collisions, to the lattice [2]. During the $\mathrm{N}$ atom studies described in reference [1], $\mathrm{H}$ atoms were examined briefly [3]. Under some circumstances they were also found to be incompletely magnetized. EPR signal enhancements of the order 1.3 were observed on addition of trace amounts of $\mathrm{O}_{2}$. Recently, this enhancing effect on the $\mathrm{H}$ atom spectrum has been reported by Breen and Glass [4] for trace additions of $\mathrm{NO}_{2}$. Westenberg and De Haas [5] have also reported an enhancement of $\mathrm{H}$ atom signals by $\mathrm{O}_{2}$, but the effect was very small and noticeable only at very high carrier flow

\footnotetext{
${ }^{1}$ Figures in brackets indicate the literature references at the end of this paper.
}

rates. Using power saturation methods, they measured $T_{1}$ for $\mathrm{H}$ and found values of the order of $100 \mu \mathrm{s}$. These are much shorter relaxation times than those observed in reference [1] for $\mathrm{N}$ atoms. Because their observed $T_{1}$ values were independent of carrier flow velocity and increased with increasing carrier pressure, they concluded that the predominant relaxation process occurred on the flow tube walls.

The work of Westenberg and De Haas prompted the writer to make a more detailed study of spinrelaxation effects on the $\mathrm{H}$ atom spectrum than that reported earlier. Both experimental and theoretical aspects of the problem were examined. For the experiments, $\mathrm{H}$ atoms were generated by thermal dissociation [6] of pure $\mathrm{H}_{2}$ thereby avoiding the need for impurities (such as $\mathrm{O}_{2}, \mathrm{~N}_{2}$, or $\mathrm{H}_{2} \mathrm{O}$ ) [7] normally required for efficient dissociation by a discharge. In this system traces of $\mathrm{O}_{2}$ added downstream from the dissociation point produced significant enhancements of the $\mathrm{H}$ atom EPR signal. Furthermore, the amount of microwave power required to produce a given degree of saturation increased with increasing $\mathrm{H}_{2}$ flow rate. These two observations demonstrated that in the absence of added $\mathrm{O}_{2}, T_{1}$ for this system was of the order of $10 \mathrm{~ms}$, far larger than that observed in the system of Westenberg and De Haas. A simplified analysis of the $\mathrm{H}-\mathrm{H}$ spin-exchange process showed that spinexchange collisions alone can lead to partial magnetization of the atoms in the absence of any added paramagnetic impurity. In a typical EPR experiment, the maximum $\mathrm{H}$ atom signal enhancement to be expected on addition of $\mathrm{O}_{2}$ is only a factor of two, in contrast to the case of $\mathrm{N}$ atoms where much larger enhancements are possible. The effects of atom diffusion on the apparent relaxation time measured by power saturation were also examined theoretically. 
Because of the difficulty in applying corrections for diffusion and the additional problems caused by inhomogeneously broadened lines and the uncertainties in estimating the microwave magnetic field strength inside the flow tube, no attempt was made to measure absolute relaxation times by power saturation methods.

\section{Theory}

Hydrogen atoms in their ground electronic state possess four magnetic hyperfine levels. In this section a.general expression will be derived for the time rate of change of the concentrations $c_{i}$ of these levels in a cylindrical flow tube.

A number of processes produce changes in the $c_{i}$. The most important ones are, (a) spin-exchange collisions between the $\mathrm{H}$ atoms themselves, (b) spin-exchange collisions with gas phase paramagnetic impurities, (c) collisions with the walls, (d) interactions with $\mathrm{rf}$ radiation, (e) mass flow, and (f) diffusion. The effects of each of these mechanisms will be evaluated separately and the results added to give the total rate of change in the $c_{i}$. The general equation contains nonlinear terms arising from the $\mathrm{H}-\mathrm{H}$ spin-exchange process. It will be linearized by considering only small departures from equilibrium. To solve the linearized equation, further simplifications are necessary. Steady state solutions will be obtained by neglecting diffusion and assuming a uniform velocity profile for the carrier. The effects of axial diffusion will be examined by solving a rate equation for a two level model, again assuming a uniform carrier velocity.

\subsection{Spin-Exchange Interaction Between H Atoms}

Consider a collision between two $\mathrm{H}$ atoms, the first in state $\Psi_{i}$ and the second in state $\Psi_{j}$. We want to calculate the probability of the first atom being in state $\Psi_{k}$ and the second in state $\Psi_{l}$ after the collision. In terms of strong field spin eigenfunctions, the wave functions $\Psi_{i}, i=1 \rightarrow 4$, for any value of the dc EPR magnetic field, are

$$
\begin{aligned}
& \Psi_{1}=\alpha a \\
& \Psi_{2}=(1-\epsilon)^{1 / 2} \alpha b+\epsilon^{1 / 2} \beta a \\
& \Psi_{3}=\beta b \\
& \Psi_{4}=(1-\epsilon)^{1 / 2} \beta a-\epsilon^{1 / 2} \alpha b
\end{aligned}
$$

where $\alpha$ and $a$ are the electron and proton spin functions respectively, whose angular momentum projections in the direction of the dc field have the value $\frac{1}{2}$, and $\beta$ and $b$ are the functions with projections $-\frac{1}{2} ; \epsilon=\left[1-x\left(x^{2}+1\right)^{-1 / 2}\right] / 2 ; x=\left(1-g_{J} / g_{P}\right)\left(\nu_{P} / \nu_{H}\right)$; $g_{J}$ and $g_{P}$ are the electron and proton $g$ factors; $\nu_{H}$ is the zero field hyperfine splitting frequency and $\nu_{P}=-g_{P} \mu_{0} H_{0} / \hbar ; \mu_{0}$ is the Bohr magneton and $H_{0}$ the magnitude of the dc field. The energy levels $U_{i}$ are given by the Breit-Rabi equation [8] and lie in the order $U_{1}>U_{2}>U_{3}>U_{4}$ for a nonzero field.
Following the treatment of Purcell and Field [9], we express the initial spin wave function for the two atoms as the product $\Psi_{i}(1) \Psi_{j}(2)$. This can be written as a superposition of singlet and triplet electron spin functions. For example, one might have

$$
\begin{aligned}
& \Psi_{\text {initial }}=\Psi_{1}(1) \Psi_{2}(2)=(1-\epsilon)^{1 / 2} \alpha(1) \alpha(2) a(1) b(2) \\
& +1 / 2 \epsilon^{1 / 2}[\alpha(1) \beta(2)+\beta(1) \alpha(2)] a(1) a(2) \\
& \quad+1 / 2 \epsilon^{1 / 2}[\alpha(1) \beta(2)-\beta(1) \alpha(2)] a(1) a(2) .
\end{aligned}
$$

The difference in the scattering potential for the singlet and triplet components produces a phase shift $\varphi_{t s}$ between the amplitudes of the two components. This phase shift is given by the equation $\varphi_{t s}=\int\left(V_{t}-V_{s}\right) d t / \hbar$, where $V_{t}$ and $V_{s}$ are the energies of the triplet and singlet electronic states, and the integration is over the duration of the collision.

After the collision the wave function becomes,

$$
\begin{aligned}
\Psi_{\text {final }}= & (1-\epsilon)^{1 / 2} \alpha(1) \alpha(2) a(1) b(2) \\
& +1 / 2 \epsilon^{1 / 2}[\alpha(1) \beta(2)+\beta(1) \alpha(2)] a(1) a(2) \\
& +1 / 2 \epsilon^{1 / 2}[\alpha(1) \beta(2)-\beta(1) \alpha(2)] a(1) a(2) \exp \left(i \varphi_{t s}\right) .
\end{aligned}
$$

The amplitude of the contribution of a particular product function $\Psi_{k}(1) \Psi_{l}(2)$ to $\Psi_{\text {final }}$ is given by the integral $\int \Psi_{k}(1) \Psi_{l}(2) \Psi_{\text {final }} d \tau$, where the integration is over all space. The square of this amplitude gives the probability that after the collision the state $\Psi_{\text {initial }}$ becomes the state $\Psi_{k}(1) \Psi_{l}(2)$.

Performing this calculation for all possible combinations of initial product functions $\Psi_{i}(1) \Psi_{j}(2)$ and all possible final product functions $\Psi_{k}(1) \Psi_{t}(2)$, one obtains a $16 \times 16$ matrix of transition probabilities. Because of the small energy differences between the hyperfine levels this matrix is not quite symmetric. By detailed balancing, the rate of the transition $i j \rightarrow k l$, at equilibrium, must equal the rate of the reverse process $k l \rightarrow i j$. This can happen only if the matrix elements satisfy the relation $(k l, i j)=$ $(i j, k l) \exp \left[\left(U_{k}+U_{l}-U_{i}-U_{j}\right) / k T\right]$, where $(i j, k l)$ is the probability for the transition $i j \rightarrow k l, k$ is Boltzmann's constant, and $T$ is the gas kinetic temperature. The error involved in calculating the transition probabilities in this manner is discussed by Purcell and Field. It is of the order $\left(U_{k}+U_{l}-U_{i}-U_{j}\right) / k T$ and thus quite small.

The nonzero elements of this matrix are given in table 1 as a function of the field strength parameter $\epsilon$ and a phase shift parameter $\Delta=\frac{1}{2} \sin ^{2}\left(\varphi_{t s} / 2\right)$. Only strong collisions for which $\varphi_{t s} \gg 1$ will be considered. In this case $\Delta=\frac{1}{4}$. Transition probabilities for the zero field case treated by Purcell and Field can be obtained from table 1 by letting $\epsilon=\frac{1}{2}$. For an EPR absorption at $\mathrm{X}$ band frequencies, $\epsilon \sim 0.5 \times 10^{-2}$ for the high field $\mathrm{H}$ atom line. The matrix element $(i j, k l)$ is only the relative probability that $i j \rightarrow k l$ in a spin-exchange collision. The overall rate at which such collisions 
occur will be specified by a rate constant $S$. A literature value will be used for this parameter.

The rates of change of the $c_{i}$ are given by

$$
\begin{aligned}
\dot{c}_{i} S^{-1}= & -\sum_{j} c_{i} c_{j}-\sum_{j \neq i} c_{i} c_{j} \\
& +\sum_{k, l} c_{k} c_{l}\left\{\sum_{m}(k l, i m)+\sum_{m \neq i}(k l, m i)\right\} \\
& =\sum_{k, l} a_{k l i} c_{k} c_{l}, \quad i=1 \rightarrow 4
\end{aligned}
$$

where the coefficients $a_{k l i}$ are various linear combinations of the matrix elements $(i j, k l)$. These equations represent changes in the $c_{i}$ arising from spin-exchange collisions between the $\mathrm{H}$ atoms themselves. Let us now consider changes resulting from interactions of the atoms with the external system.

\begin{tabular}{|c|c|}
\hline Transition $^{\mathrm{a}}$ & Transition probability \\
\hline $\begin{array}{l}(12,14),(12,41) \\
(13,22),(13,44) \\
(14,21) \\
(21,41) \\
(22,31) \\
(23,34),(23,43) \\
(31,44) \\
(32,34),(32,43)\end{array}$ & $\epsilon(1-\epsilon) \Delta$ \\
\hline $\begin{array}{l}(12,21) \\
(13,24) \\
(34,43)\end{array}$ & $\epsilon^{2} \Delta$ \\
\hline $\begin{array}{l}(13,42) \\
(14,41) \\
(23,32)\end{array}$ & $(1-\epsilon)^{2} \Delta$ \\
\hline $\begin{array}{l}(22,24),(22,42) \\
(24,44) \\
(42,44)\end{array}$ & $\epsilon(1-\epsilon)(1-2 \epsilon)^{2} \Delta$ \\
\hline $\begin{array}{l}(22,44) \\
(24,42)\end{array}$ & $4 \epsilon^{2}(1-\epsilon)^{2} \Delta$ \\
\hline$(11,11),(33,33)$ & 1 \\
\hline $\begin{array}{l}(12,12),(21,21) \\
(34,34),(43,43)\end{array}$ & $1-2 \epsilon\left(1-\frac{1}{2} \epsilon\right) \Delta$ \\
\hline$(13,13),(31,31)$ & $1-\Delta$ \\
\hline $\begin{array}{l}(14,14),(41,41) \\
(32,32),(23,23)\end{array}$ & $1-\left(1-\epsilon^{2}\right) \Delta$ \\
\hline$(22,22),(44,44)$ & $\begin{aligned} 1-4 \epsilon & (1-\epsilon) \\
& {[1-\epsilon(1-\epsilon)] \Delta }\end{aligned}$ \\
\hline$(24,24),(42,42)$ & $1-\left[1-4 \epsilon^{2}(1-\epsilon)^{2}\right] \Delta$ \\
\hline \multicolumn{2}{|c|}{$(k l, i j)=(i j, k l) \exp \left[\left(U_{k}+U_{1}-U_{i}-U_{j}\right) / k T\right]$} \\
\hline
\end{tabular}

TABlE 1. Nonzero transition probabilities for spin-exchange collisions between $H$ atoms, $(\mathrm{ij}, \mathrm{kl})$.

a The matrix element $(i j, k l)$ corresponds to the transition $\psi_{i}(1) \psi_{j}(2) \rightarrow \psi_{k}(1) \psi_{l}(2)$, where the numbers in parentheses label the two atoms involved in the collision.

\subsection{Spin-Lattice Interactions}

For $\mathrm{H}$ atoms in a nonpolar, nonparamagnetic carrier, the only important spin-lattice relaxation processes are spin-exchange collisions with paramagnetic impurities and wall collisions. The paramagnetic impurity is assumed to have orbital angular momentum so that its electron spin will be strongly coupled to the lattice. The spin temperature of this species will thus always remain at the temperature of the lattice [2]. We shall not try to specify the details of the impurity interactions, but shall simply let $K_{i j}$ be an overall rate constant for a particular transition $\Psi_{i} \rightarrow \Psi_{j}$ produced by these interactions.

The total rate of change in the $c_{i}$ resulting from these transitions will be

$$
\dot{c}_{i}=\sum_{k \neq i}\left(K_{k i} c_{k}-K_{i k} c_{i}\right)=\sum_{j} R_{i j} c_{j}
$$

where $R_{i j}=K_{j i}$ and $R_{i i}=-\sum_{j \neq i} K_{i j}$. From detailed balancing we have $K_{i j}=K_{j i} \exp \left[\left(U_{i}-U_{j}\right) / k T\right]$. One would expect the $K_{i j}$ to be proportional to the concentration of a particular paramagnetic impurity and to depend on its nature.

Relaxation by wall collisions also yields terms which are first order in the $c_{i}$. If these were treated together with the gas phase interactions a single set of $K_{i j}$ parameters could be used to represent both processes. It will be shown later that the wall relaxation process can be included explicitly by treating the interaction as a boundary condition on the rate equation when radial diffusion of the atoms is included.

\subsection{Transitions Induced by an if Magnetic Field}

The rate of change in the $c_{i}$ arising from absorption and induced emission by an rf magnetic field is given

$$
\dot{c}_{i}=\sum_{k \neq i}\left(W_{k i} c_{k}-W_{i k} c_{i}\right)=\sum_{j} w_{i j} c_{j}
$$

where the $W_{i j}$ are rate constants for these transitions, $w_{i j}=W_{j i}$, and $w_{i i}=-\sum_{j \neq i} W_{i j}$. In the present experiment, the high field $\mathrm{H}$ atom line which arises from transitions between levels 2 and 3 was examined. For this case all $W_{i j}=0$ except $W_{23}$ and $W_{32}$ which are equal. Let $W_{23}$ and $W_{32}$ equal $W$. The rate constant $W$ is proportional to the rf power striking the sample. Because the rf field varies from point to point throughout the microwave cavity, $W$ will be different at different points ' in the cavity. We have $W=h^{-2} H_{1}^{2} \mu^{2} g(\nu)$, where $H_{1}$ is the amplitude of the rf magnetic field, $\mu^{2}$ is the square of the absolute value of the transition matrix element, and $g(\nu)$ is the normalized line shape function [10].

\subsection{Effects of Mass Flow and Diffusion}

To complete the rate equations for $c_{i}$, one must include a term which accounts for the flow of atoms 
through the microwave cavity and another which represents their diffusion. The experimental arrangement consisted of a cylindrical flow tube running through a $T E_{011}$ cavity with its axis coinciding with that of the cavity. Were relaxation on the walls as effective a 1 in 10 collisions in the present experiments, large radial gradients would arise in the population differences between the levels of interest during a saturation experiment. In this case both radial and axial diffusion terms should be included.

\subsection{Complete Rate Equations and Their Linearization}

The complete rate equations for the $c_{i}$ are, in cylindrical coordinates

$$
\begin{aligned}
\frac{\partial c_{i}}{\partial t}=S \sum_{k, l} a_{k l i} c_{k} c_{l}+\sum_{j} R_{i j} c_{j}+\sum_{j} w_{i j} c_{j} \\
-v(r) \frac{\partial c_{i}}{\partial z}+D\left\{\frac{1}{r} \frac{\partial}{\partial r}\left(r \frac{\partial c_{i}}{\partial r}\right)+\frac{\partial^{2} c_{i}}{\partial z^{2}}\right\}
\end{aligned}
$$

where $v(r)=2 v_{0}\left[1-(r / a)^{2}\right]$ the carrier flow velocity, $v_{0}$ is the average carrier velocity, $a$ is the radius of the flow tube, and $D$ is the atom's diffusion coefficient at the prevailing carrier pressure. The first term in the brackets represents radial diffusion and the second axial diffusion. The term containing $v(r)$ accounts for the effects of mass flow. For reasons discussed earlier, the coefficients $w_{i j}$, which represent the effect of $\mathrm{rf}$ power, are functions of $r$ and $z$. The sum containing the parameters $R_{i j}$ represents the effect of interactions with paramagnetic impurities and if desired, the effect of relaxation on the walls. The first sum represents the effect of spin-exchange collisions among the $H$ atoms themselves.

Under all conditions experienced in a typical EPR experiment with $\mathrm{H}$ atoms, the $c_{i}$ differ at most by several parts per thousand. The rate equations therefore can be linearized [11] by expressing the $c_{i}$ in the form $c_{i}=\frac{1}{4} n\left(1+\xi_{i}\right)$, where $n$ is the total $\mathrm{H}$ atom concentration and the $\xi_{i}$ are very small numbers which will be considered only to first order. As a result of this definition, the relation $\sum_{i} \xi_{i}=0$ must always be satisfied.

With this substitution, the rate equations become.

$$
\begin{aligned}
& \frac{\partial \xi_{i}}{\partial t}=\frac{1}{4} S n\left\{\sum_{k, l} a_{k l i}+\sum_{j} A_{i j} \xi_{j}\right\}+\sum_{j} R_{i j}\left(1+\xi_{j}\right) \\
& +\sum_{j} w_{i j} \xi_{j}-v(r) \frac{\partial \xi_{i}}{\partial z}+D\left\{\frac{1}{r} \frac{\partial}{\partial r}\left(r \frac{\partial \xi_{i}}{\partial r}\right)+\frac{\partial^{2} \xi_{i}}{\partial z^{2}}\right\}
\end{aligned}
$$

where the relation $\sum_{j} w_{i j}=0$ has been used. The $A_{i j}$ are linear combinations of the $a_{k l i}$, which in turn are linear combinations of the transition probabilities $(i j, k l)$ from table 1.

The Boltzmann factors in the $(i j, k l)$ and in the rate constants $K_{i j}$ from which the $R_{i j}$ are derived ensure that these rate equations will satisfy the proper equilibrium conditions. However, the equations are easier to handle if they are rewritten so that the equilibrium values of the $\xi_{i}$ are included explicitly. This may be done in the following manner. For the system at thermal equilibrium, with no $\mathrm{rf}$ power applied, the rate equations become

$$
\frac{1}{4} S n \sum_{k, l} a_{k l i}+\sum_{j} R_{i j}=-\frac{1}{4} S n \sum_{j} A_{i j} E_{j}-\sum_{j} R_{i j} E_{j}
$$

where the $E_{i}$ are the equilibrium values of $\xi_{i}$ for the particular lattice temperature and de field.

Substituting this expression into (6) gives

$$
\begin{aligned}
& \frac{\partial \xi_{i}}{\partial t}=\frac{1}{4} S n \sum_{j} A_{i j}\left(\xi_{j}-E_{j}\right)+\sum_{j} R_{i j}\left(\xi_{j}-E_{j}\right) \\
& +\sum_{j} w_{i j} \xi_{j}-v(r) \frac{\partial \xi_{i}}{\partial z}+D\left\{\frac{1}{r} \frac{\partial}{\partial r}\left(r \frac{\partial \xi_{i}}{\partial r}\right)+\frac{\partial^{2} \xi_{i}}{\partial z^{2}}\right\} .
\end{aligned}
$$

In this form the equations give the correct equilibrium values of $\xi_{i}$ even if the Boltzmann factors inherent in the $A_{i j}$ and $R_{i j}$ are neglected. The error in doing so will be very small since the factors are so close to unity.

\subsection{Evaluation of the Coefficients $A_{i j}$ and $R_{i j}$}

The coefficients $A_{i j}$ which account for the effects of $H-H$ spin-exchange collisions are linear combinations of the transition probabilities $(i j, k l)$ shown in table 1. They are given in table 2 in the form of a $4 \times 4$ matrix which is valid for all values of the dc field. Strong collisions, for which $\Delta=\frac{1}{4}$, have been assumed, and the Boltzmann factors neglected. In this table an additional field strength parameter, $\chi=8 \epsilon(1-\epsilon)$ has been introduced. Lambe [11] has also determined the $A_{i j}$ coefficients by a somewhat different method.

In the strong field limit, the coefficients $R_{i j}$ which describe the spin-lattice interactions can easily be derived if we consider only interactions which change the electron spin state. These interactions could be with a gaseous paramagnetic impurity or with the walls of the flow tube. On changing the electron spin quantum number, the strong field wave functions for

TABLE 2. The coefficients $\mathrm{A}_{\mathrm{ij}}$ which describe the effects of $\mathrm{H}-\mathrm{H}$ spinexchange collisions.

The parameter $\chi$ equals $8 \epsilon(1-\epsilon)$

\begin{tabular}{cc|r|r|r|c}
\hline \hline$i$ & $j$ & 1 & 2 & 3 & 4 \\
\hline 1 & -1 & 1 & -1 & 1 \\
2 & 1 & $-(1+\chi)$ & 1 & $-(1-\chi)$ \\
3 & -1 & 1 & -1 & 1 \\
4 & 1 & $-(1-\chi)$ & 1 & $-(1+\chi)$
\end{tabular}


the four hyperfine states transform in the following way:

$$
\begin{aligned}
& \Psi_{1}=\alpha a \rightarrow \beta a=\Psi_{4} \\
& \Psi_{2}=\alpha b \rightarrow \beta b=\Psi_{3} \\
& \Psi_{3}=\beta b \rightarrow \alpha b=\Psi_{2} \\
& \Psi_{4}=\beta a \rightarrow \alpha a=\Psi_{1} .
\end{aligned}
$$

The rates of change in the $c_{i}$ produced by this interaction are thus

$$
\begin{aligned}
& \dot{c}_{1}=K_{41} c_{4}-K_{14} c_{1} \\
& \dot{c}_{2}=K_{32} c_{3}-K_{23} c_{2} \\
& \dot{c}_{3}=K_{23} c_{2}-K_{32} c_{3} \\
& \dot{c}_{4}=K_{14} c_{1}-K_{41} c_{4} .
\end{aligned}
$$

where $K_{i j}=\frac{1}{2} K$, and $K$ is an overall rate constant for these interactions. The reason why the factor $\frac{1}{2}$ has been introduced will be discussed later. The $R_{i j}$ can be derived from the $K_{i j}$ by means of (3).

By considering only the strong field limit, one is assuming that the spin-lattice interaction effects only the electron spin. Although this leads to equilibration of the population differences $c_{1}-c_{4}$ and $c_{2}-c_{3}$ which are the ones involved in the EPR transitions, it will not by itself produce thermal equilibration of the $c_{i}$. This is because transitions between the states $\Psi_{1}, \Psi_{4}$ and $\Psi_{2}, \Psi_{3}$ have been neglected. Such transitions involve changes in the proton spin state. One way to estimate the relative $K_{i j}$ for such changes is through an adaptation of the method used by Lambe [11] to determine the rate equations for $\mathrm{H}-\mathrm{H}$ spin-exchange collisions. Consider the paramagnetic impurities to be a reservoir of electron spins in which $\mathrm{P}^{+}$and $\mathrm{P}^{-}$are the probabilities of finding an electron with spin up and spin down, respectively. Since the impurity spins are assumed to be strongly coupled to the lattice, and thus always at thermal equilibrium, $P^{+}$and $P^{-}$will be constant and have almost the same value, namely $\frac{1}{2}$, at the temperatures and field strengths used in the present experiments. Suppose that an $\mathrm{H}$ atom in state $\Psi_{1}$ exchanges its electron with one from the impurity reservoir. With probability $P^{+}, \Psi_{1}$ remains unchanged. With probability $P^{-}, \Psi_{1}$ goes to state $\beta a$ which is a mixed state. We have

$$
\beta a=(1-\epsilon)^{1 / 2} \Psi_{4}+\epsilon^{1 / 2} \Psi_{2} .
$$

so that with a probability $\epsilon P^{-}, \Psi_{1}$ goes to $\Psi_{2}$, and with a probability $(1-\epsilon) P^{-}$, to $\Psi_{4}$.

Following this procedure for all the states $\Psi_{i}$, one gets rate equations for the $c_{i}$. For example,

$$
\begin{aligned}
\dot{c}_{1} & =-K P^{-} c_{1}+K \epsilon P^{+} c_{2}+K(1-\epsilon) P^{+} c_{4} \\
& =-\frac{1}{2} K c_{1}+\frac{1}{2} K \epsilon c_{2}+\frac{1}{2} K(1-\epsilon) c_{4}
\end{aligned}
$$

where $K$ is an overall rate constant for spin-exchange collisions between the $\mathrm{H}$ atoms and the paramagnetic impurity. It will be proportional to the impurity concentration.

On making the transformation $c_{i}=\frac{1}{4} n\left(1+\xi_{i}\right)$, and introducing the equilibrium parameters $E_{i}$, one obtains the rate equations in the desired form $\dot{\xi}_{i}$ $=\sum_{j} R_{i j}\left(\xi_{j}-E_{j}\right)$, where the matrix $R_{i j}$ is given in table 3 . For the strong field case, $\epsilon \rightarrow 0$, and the $R_{i j}$ reduce to the values obtained earlier. The factor $\frac{1}{2}$ in these coefficients arises because $\frac{1}{2}$ of the impurity spins in the strong field case will be in the same direction as the electron spin on the $\mathrm{H}$ atom and so no change in the state of $\mathrm{H}$ will occur during an electron exchange in one out of two collisions.

While the matrix $R_{i j}$ in table 3 should be valid for all field strengths, only the strong field elements were used to analyze the experimental data. The $\mathrm{H}-\mathrm{H}$ spinexchange interactions also produce the transitions neglected in the strong field spin-lattice approximation. Since the $\mathrm{H}-\mathrm{H}$ exchange dominates the spin-lattice interactions in the present experiments, the spinlattice contributions to these transitions can be ignored.

TABLE 3. The coefficients $\mathrm{R}_{i j}$, which describe the spin-lattice interactions.

\begin{tabular}{c|c|c|c|c}
\hline \multicolumn{6}{c}{ The parameter $\chi$ equals $8 \epsilon(1-\epsilon)}$. \\
\hline$j$ & 1 & 2 & 3 & 4 \\
\hline 1 & & & & \\
\hline 2 & $-\frac{1}{2} K$ & $\frac{1}{2} \epsilon K$ & 0 & $\frac{1}{2}(1-\epsilon) K$ \\
3 & $\frac{1}{2} \epsilon K$ & $-\frac{1}{2}\left(1+\frac{1}{4} \chi\right) K$ & $\frac{1}{2}(1-\epsilon) K$ & $\chi K / 8$ \\
4 & $\frac{1}{2}(1-\epsilon) K$ & $\frac{1}{2}(1-\epsilon) K$ & $-\frac{1}{2} K$ & $\begin{array}{c}\frac{1}{2} \epsilon K \\
2\end{array}$ \\
\hline & & $\chi K / 8$ & $\frac{1}{2} \epsilon K$ & $-\frac{1}{2}\left(1+\frac{1}{4} \chi\right) K$ \\
\hline
\end{tabular}

\subsection{Wall Relaxation as a Boundary Condition}

If relaxation on the walls were rapid enough to produce radial concentration gradients, the rate equation (7) should be solved subject to the boundary conditions

$$
\left\{\frac{\partial \xi_{i}}{\partial r}\right\}_{r=a}=-\frac{1}{2} a D \sum_{j}\left(R_{w}\right)_{i j}\left(\xi_{j}-E_{j}\right)
$$

where the derivatives are evaluated at the wall of the flow tube, $D$ is the diffusion coefficient for the atom in the carrier gas at the prevailing carrier pressure, and the $\left(R_{w}\right)_{i j}$ are coefficients for wall relaxation analogous to the $R_{i j}$ defined for relaxation by paramagnetic impurities in the gas phase. In the present experiments wall relaxation cannot have been rapid. Therefore the radial diffusion term in (7) will be dropped, and any wall relaxation effects will be absorbed in the $R_{i j}$ coefficients instead of being included explicitly as a boundary condition.

\subsection{A Two-Level Spin Model}

In order to examine the effects of axial diffusion, a two-level model consisting of particles with spin $1 / 2$ was 
used. For such a system, the $A_{i j}=0$, while the matrices for $R_{i j}$ and $w_{i j}$ are simply

$$
R_{i j}=\left(\begin{array}{rr}
-\frac{1}{2} K & \frac{1}{2} K \\
\frac{1}{2} K & -\frac{1}{2} K
\end{array}\right)
$$

and,

$$
w_{i j}=\left(\begin{array}{rr}
-W & W \\
W & -W
\end{array}\right)
$$

where $K$ and $W$ are analogous to the same quantities defined for the four-level model.

\section{Apparatus and Experiments}

The apparatus used was essentially that described in reference 1 except that the klystron frequency was now locked to the cavity resonance instead of to an external oscillator. Field modulation was at $400 \mathrm{~Hz}$. Hydrogen atoms were produced outside the high field of the EPR magnet by passing Matheson* Ultra High Purity $\mathrm{H}_{2}$ over a tungsten filament [6] heated to about $1600{ }^{\circ} \mathrm{C}$. Only $\mathrm{H}_{2}$ was used as a carrier. The walls of the $10 \mathrm{~mm}$ in diam quartz flow tube were poisoned against atom recombination with boric acid [12]. The amount of atom decay was measured by adding a trace of NO to the atom stream and observing the HNO emission [13]. Between the EPR cavity and the point of $\mathrm{O}_{2}$ addition, the decay was less than 5 percent for all experiments. The length of flow tube in the high-field zone upstream from the cavity was estimated to be about $15 \mathrm{~cm}$. Impurity $\mathrm{O}_{2}$ was normally added at a point $45 \mathrm{~cm}$ downstream from the filament and $27 \mathrm{~cm}$ upstream from the cavity center in the form of a 1:500, $\mathrm{O}_{2}$ : Ar mixture. At $\mathrm{H}_{2}$ flow rates of the order $3.5 \mathrm{~atm} \mathrm{~cm}^{3} \mathrm{~s}^{-1}$, and pressures of 2 torr, a 2 -fold enhancement of the unsaturated high field $\mathrm{H}$ atom EPR line was observed on addition of $15 \mathrm{ppm}$ of $\mathrm{O}_{2}$. Oxygen additions greater than this produced no further increase in the signal, nor did the addition of this same $15 \mathrm{ppm}$ of $\mathrm{O}_{2}$ further upstream.

At constant atom concentrations in the cavity, the amount of power required to produce a given degree of saturation was found to increase with increasing carrier flow velocity. Carrier velocities from 650 to $4400 \mathrm{~cm} \mathrm{~s}^{-1}$ were covered in these saturation experiments.

Analysis of these experiments indicated that $T_{1}$ in our system was of the order of $20 \mathrm{~ms}$ in the absence of added $\mathrm{O}_{2}$, a value similar to that observed for $\mathrm{N}$ atoms. The neglect of the radial diffusion term in (7) is therefore justified, since even if a wall mechanism were the sole relaxation process, the radial gradients it would produce would be negligible at the flows and pressures used.

${ }^{2}$ Certain commercial products and instruments are identified in this paper in order to specify adequately the experimental procedure. In no case does such identification imply recommendation or endorsement by the National Bureau of Standards, nor does it imply that the products or equipment identified are necessarily the best available for the purpose.

\section{Solution of the Rate Equations}

Since a flow method was used, only steady-state solutions of the rate equations were required. The equations (7) for the four-level system were solved only under the following conditions: (a) radial and axial diffusion neglected; (b) for the unsaturated case, a uniform velocity profile for the carrier, and (c) for the saturated case, a zero carrier velocity. To examine the effects of axial diffusion at low and high power, and nonzero carrier velocity under saturation conditions, a two-level model was analyzed. Again radial diffusion was neglected, and a uniform carrier velocity assumed.

\subsection{Unsaturated Four-Level System}

For low power conditions such that the coefficients $w_{i j}$ are negligible, diffusion is neglected, and the carrier velocity is assumed to be uniform, the steadystate solution of (7) is given by the set of equations,

$$
\begin{aligned}
& \xi_{1}=E_{1}+\frac{1}{2}(V+Y) \\
& \xi_{2}=E_{2}+\frac{1}{2}(U-V) \\
& \xi_{3}=E_{3}+\frac{1}{2}(V-Y) \\
& \xi_{4}=E_{4}+\frac{1}{2}(U+V)
\end{aligned}
$$

where,

$$
\begin{aligned}
& V=V_{0} \exp \left[-2\left(2 \gamma+\frac{1}{2} K\right)\left(z / v_{0}\right)\right] \\
& Y=\frac{1}{2} \zeta^{-1}\left\{\left(Y_{0} \theta^{+-}-\frac{1}{2} K U_{0}\right) \exp \left(\Phi^{-}\right)\right. \\
& \left.+\left(Y_{0} \theta^{-}+\frac{1}{2} K U_{0}\right) \exp \left(\Phi^{+}\right)\right\} \\
& U=\frac{1}{2} \zeta^{-1}\left\{\left(U_{0} \theta^{-}-\frac{1}{2} K Y_{0}\right) \exp \left(\Phi^{-}\right)\right. \\
& \left.+\left(U_{0} \theta^{+}+\frac{1}{2} K Y_{0}\right) \exp \left(\Phi^{+}\right)\right\}
\end{aligned}
$$

and,

$$
\begin{aligned}
& \gamma=\frac{1}{4} S n \\
& \zeta=\left(\eta^{2}-K \gamma \chi\right)^{1 / 2} \\
& \eta=\frac{1}{2} K+\gamma \chi \\
& \theta^{ \pm}=\zeta \pm \gamma \chi \\
& \Phi^{ \pm}=-(\eta \pm \zeta)\left(z / v_{0}\right) .
\end{aligned}
$$

The subscript zero on the parameters $U, V$, and $Y$ denotes their values at $z=0$, the start of the high field region. As mentioned earlier, the strong field values of the $R_{i j}$ coefficients were used.

Calculated values of the $\xi_{i}$ parameters are shown in figures 1 and 2 as functions of $z / v_{0}$. For this calculation the $\xi_{i}$ values at the start of the high field region were taken to be the values corresponding to thermal equilibrium at zero field and $300 \mathrm{~K}$, namely, $\xi_{1}^{0}=\xi_{2}^{0}$ $=\xi_{3}^{0}=-0.572 \times 10^{-4}$, and $\xi_{4}^{0}=1.716 \times 10^{-4}$. An $\mathrm{H}$ atom concentration of $0.44 \times 10^{14} \mathrm{~cm}^{-3}$ and a $\mathrm{H}-\mathrm{H}$ spin- 


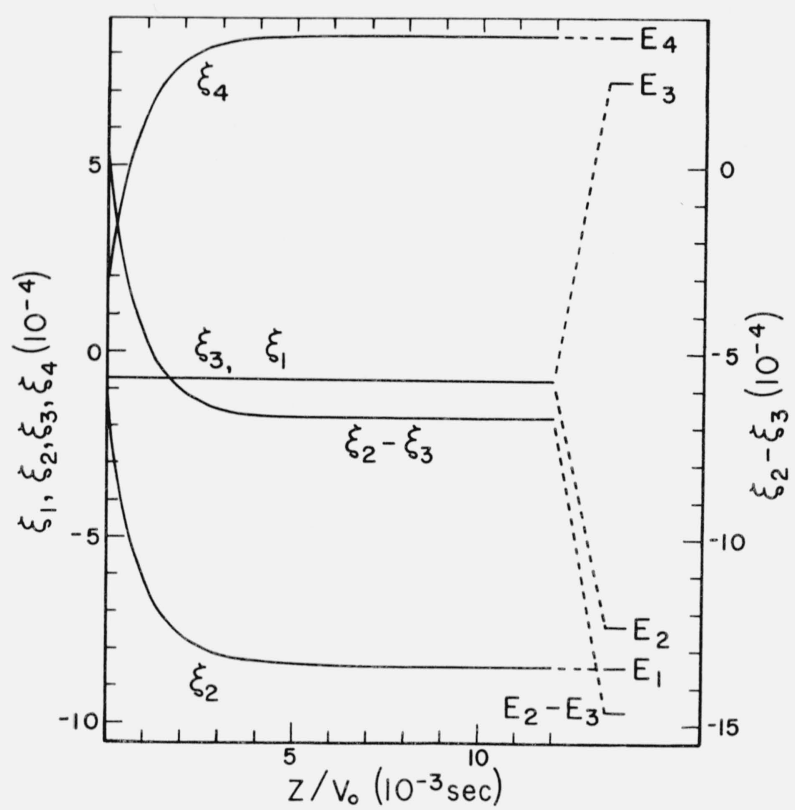

FigURE 1. Population parameters $\xi_{i}$ versus $\mathrm{z} / \mathrm{v}_{0}$ calculated from eq 9.

Parameter values used were $K=0, S=13 \times 10^{-10} \mathrm{~cm}^{3} \mathrm{~s}^{-1}, n=0.44 \times 10^{14} \mathrm{~cm}^{-3}$, dc field $=3500$ Gauss, and $T=300 \mathrm{~K}$. The difference $\xi_{2}-\xi_{3}$ is also plotted.

exchange rate constant of $13 \times 10^{-10} \mathrm{~cm}^{3} \mathrm{~s}^{-1}$ were used. In the present apparatus the EPR cavity would lie at $z / v_{0}=12 \times 10^{-3} \mathrm{~s}$ for $v_{0}=800 \mathrm{~cm} \mathrm{~s}^{-1}$. In figure 1 , the spin-lattice relaxation parameter $K$ has been set equal to zero, while in figure 2 , it was given a value of $168 \mathrm{~s}^{-1}$. Also shown in both figures is the difference $\xi_{2}-\xi_{3}$. The intensity of the high field EPR line will be proportional to the value of this difference at the cavity position. Thermal equilibrium values of the parameters at a field of 3500 Gauss and $300 \mathrm{~K}$ are shown as dotted lines.

In the absence of any spin-lattice relaxation, $\xi_{1}$ and $\xi_{3}$ remain unchanged, while $\mathrm{H}-\mathrm{H}$ spin-exchange brings about rapid relaxation of $\xi_{2}$ and $\xi_{4}$ to their equilibrium values $-8.431 \times 10^{-4}$ and $8.490 \times 10^{-4}$, respectively. They approach to within 10 percent of their final values in a time of the order $1 / 3 \gamma \chi$. Because $\chi$ is so small $(\sim 0.04)$, this is considerably slower than one might expect from the large value of $\gamma$ at this particular $\mathrm{H}$ atom concentration. It is obvious that the strong field wave functions would not be suitable for this problem because they yield $\chi=0$ and thus no relaxation of $\xi_{2}$ and $\xi_{4}$.

The absolute value of $\xi_{2}-\xi_{3}$ for this partially relaxed condition is $6.77 \times 10^{-4}$, which is a factor of 2.16 smaller than the thermal equilibrium value. Therefore, unless one operates at considerably smaller $\mathrm{H}$ atom concentrations, or at much higher flow rates, the maximum enhancement of the EPR signal to be expected on addition of a paramagnetic impurity would be this factor of 2.16.

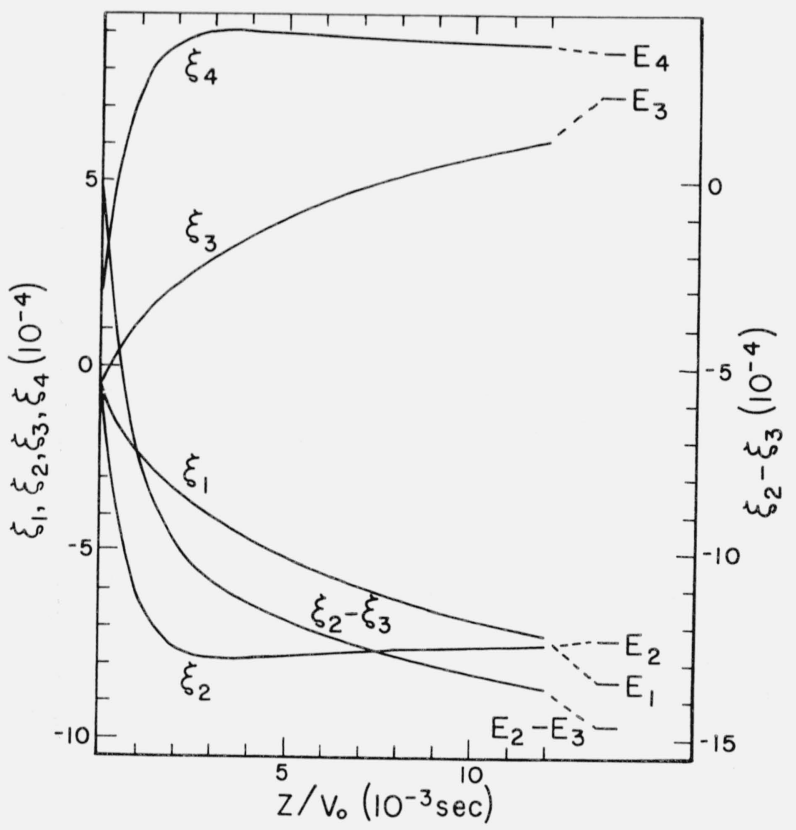

Figure 2. Population parameters $\xi_{i}$ versus $\mathrm{z} / \mathrm{v}_{0}$ calculated from eq 9 .

Parameter values used were $K=168 \mathrm{~s}^{-1}, S=13 \times 10^{-10} \mathrm{~s}^{-1}, n=0.44 \times 10^{14} \mathrm{~cm}^{-3}$, dc field $=3500 \mathrm{Gauss}$, and $T=300 \mathrm{~K}$. The difference $\xi_{2}-\xi_{3}$ is also plotted.

In case $\gamma \chi \gg K$, the solution for $\xi_{2}-\xi_{3}$ may be written approximately as

$$
\begin{array}{r}
\xi_{2}-\xi_{3}=E_{2}-E_{3}+\frac{1}{2}\left\{Y_{0}-U_{0} \exp \left(-2 \gamma \chi z / v_{0}\right)\right\} \\
\exp \left(-\frac{1}{2} K z / \nu_{0}\right) .
\end{array}
$$

The term in brackets quickly relaxes to the value $Y_{0}$ so that the process can be described by a single relaxation rate over the range of slow decay.

Let us now assume that $K$ may be written as a sum of two terms, i.e., $K=K_{0}+Q\left[\mathrm{O}_{2}\right]$, where $Q$ is a rate constant for spin-exchange collisions between $\mathrm{H}$ and $\mathrm{O}_{2},\left[\mathrm{O}_{2}\right]$ is the $\mathrm{O}_{2}$ concentration, and $K_{0}$ is the value of $K$ without added $\mathrm{O}_{2}$, presumably arising from collisions with paramagnetic impurities and/or wall relaxation. From the observed values of the high-field line intensity as a function of added $\mathrm{O}_{2}$, and the results of calculated $\xi_{i}$ like those shown in figures 1 and 2 , a value of $6.4 \pm 2.0 \times 10^{-10} \mathrm{~cm}^{3} \mathrm{~s}^{-1}$ (corrected for axial diffusion as described in the next section) was found for $Q$, and a value of $50 \pm 20 \mathrm{~s}^{-1}$ for $K_{0}$ at a $\mathrm{H}_{2}$ flow of $3.5 \mathrm{~atm} \mathrm{~cm}^{3} \mathrm{~s}^{-1}$ and a pressure of 2.2 torr. No measurements were made at other flows or pressures.

\subsection{Unsaturated Two-Level System and Corrections for Axial Diffusion}

For a two level system, a steady-state analytical solution of (7) is easily obtained if radial diffusion is neglected and a uniform carrier velocity is assumed. 
The solution for the population difference $\delta \xi$ between the two levels in the unsaturated case is

$$
\delta \xi=\delta E+\left(\delta \xi^{0}-\delta E\right) \exp \left\{\frac{v_{0}-\left(v_{0}^{2}+4 K D\right)^{1 / 2}}{2 D} z\right\}
$$

where $\delta E$ is $\delta \xi$ at equilibrium and $\delta \xi^{0}$ is $\delta \xi$ at $z=0$, the start of the high field region; $\delta \xi^{0}$ will be assumed to be zero.

If $v_{0}^{2} \gg 4 K D$, the solution may be approximated as

$$
\delta \xi \sim \delta E+\delta E \exp \left\{-K\left[1-\left(K D / v_{0}^{2}\right)\right]\left(z / v_{0}\right)\right\} .
$$

This result was used to estimate the effects of axial diffusion in the four-level system. For the $\mathrm{O}_{2}$ signalenhancement experiment discussed in the preceding section, the correction for this axial diffusion to the rate constant $Q$ was small, approximately +10 percent.

\subsection{Four-Level System With Saturation}

Consider equations (7) at high microwave power levels where the factors $w_{i j}$ cannot be neglected. For a nonflowing gas, without diffusion, the steady-state solution for the population difference of interest is

$$
\xi_{2}-\xi_{3}=\left(E_{2}-E_{3}\right)\left(1+T_{1} W\right)^{-1}
$$

where $T_{1}$ is given by the expression

$$
T_{1}=K^{-1}(K / \gamma+2)\left(\frac{1}{2} K / \gamma+2\right)^{-1} \text {. }
$$

This parameter is equivalent to the reciprocal of the relaxation probability parameter $W_{r}$ defined by Lloyd and Pake [10]. The factor multiplying $K^{-1}$ ranges between the limits 1 and 2 according to whether the ratio $K / \gamma$ is small or large. Although $K / \gamma$ is much less than unity in the present experiments, large $\mathrm{O}_{2}$ additions can easily make $K \gg \gamma$. In this situation, $T_{1}$ will not be strictly proportional to $\left[\mathrm{O}_{2}\right]^{-1}$. It also is evident that when $K / \gamma \sim 1, T_{1}$ will be somewhat dependent, through $\gamma$, on the $\mathrm{H}$ atom concentration.

\subsection{Two-Level System With Saturation}

To estimate the effect of flow and axial diffusion on the amount of microwave power required to produce a given degree of saturation, the two-level model has been used. The parameter $W$ has been assumed to be constant over the effective length $L$ of the cavity $(\sim 2 \mathrm{~cm})$ and zero elsewhere. The spin-lattice parameter $K$ has been assumed to be large enough to equilibrate the spins by the time the atoms reach the cavity. The EPR signal will be proportional to the average value of the population difference $\delta \xi$ in the cavity. This average over the length of the cavity is given by

$$
\langle\delta \xi\rangle=F^{+}+F^{-}+\frac{1}{2} K \delta E\left(\frac{1}{2} K+W\right)^{-1}
$$

where

$$
\begin{gathered}
F^{ \pm}=2\left(C^{ \pm} / \mu^{ \pm} L\right) \sinh \left(\mu^{ \pm \frac{1}{2}} L\right) \\
C^{ \pm}= \pm W \delta E\left(\frac{1}{2} K+W\right)^{-1}\left\{\left(\mu^{\mp}-\mu_{0}^{+}\right) \mu_{0}^{-} \exp \left(-\mu^{ \pm \frac{1}{2}} L\right)\right. \\
\left.-\left(\mu^{\mp}-\mu_{0}^{-}\right) \mu_{0}^{+} \exp \left(\mu^{\mp} \frac{1}{2} L\right)\right\}\left(G^{+}+G^{-}\right)^{-1} \\
G^{ \pm}= \pm\left(\mu^{+}-\mu_{0}^{ \pm}\right)\left(\mu^{-}-\mu_{0}^{\mp}\right) \exp \left[ \pm\left(\mu^{+}-\mu^{-}\right) \frac{1}{2} L\right] \\
\mu^{ \pm}=v_{0} / 2 D \pm \lambda \\
\mu_{0}^{ \pm}=v_{0} / 2 D \pm \lambda_{0} \\
\lambda=\left[v_{0}^{2}+8 D\left(\frac{1}{2} K+W\right)\right]^{1 / 2} / 2 D \\
\lambda_{0}=\left[v_{0}^{2}+8 D \frac{1}{2} K\right]^{1 / 2} / 2 D .
\end{gathered}
$$

This rather formidable expression was used to calculate $\langle\delta \xi\rangle$ as a function of $W$ for various values of the parameters, $v_{0}, D$, and $K$. It was found that the relationship between $\langle\delta \xi\rangle$ and $W$ could be represented to a very good approximation by the expression

$$
\langle\delta \xi\rangle=\delta E\left(1+T_{1}^{*} W\right)^{-1}
$$

where $T_{1}^{*}$ depends on $v_{0}, D$, and $K$.

The relaxation time for the four-level model at high $\mathrm{H}$ atom concentrations will actually be equal to $\frac{1}{2} T_{1}^{*}$. This is because $\mathrm{H}-\mathrm{H}$ spin-exchange collisions couple in the two unpumped levels which are also experiencing the same spin-lattice relaxation processes.

Some examples of how $\frac{1}{2} T_{1}^{*}$ depends on the various parameters are shown in figures 3 and 4 . Figure 3 shows the variation of $2 / T_{1}^{*}$ with $D$ for a range of $K$ values from 100 to $1600 \mathrm{~s}^{-1}$. The flow velocity $v_{0}$ was varied over the range 100 to $500 \mathrm{~cm} \mathrm{~s}^{-1}$. Over this interval the dependence on $v_{0}$ was small and is indicated by the width of the curves. It is evident that the effect of diffusion can be quite large even in the absence of any wall relaxation.

The variation of $2 / T_{1}^{*}$ with $v_{0}$ at $D=912 \mathrm{~cm}^{2} \mathrm{~s}^{-1}$ is shown is figure 4 for different values of $K$. It can be seen that for $K$ values above $400 \mathrm{~s}^{-1}$, the dependence of $2 / T_{1}^{*}$ on $v_{0}$ is quite small for $v_{0}$ in the range 0 to 1000 $\mathrm{cm} \mathrm{s}^{-1}$.

\subsection{Determination of $T_{1}$ by Saturation Methods}

The fact that the population differences of the pumped levels for the models considered here are proportional to a quantity of the form $\left(1+T_{1} W\right)^{-1}$ means that the saturation theory for Lorentzian lines discussed by Westenberg and De Haas [5] is applicable. Caution must be observed in using this theory, however, as it applies only to homogeneously broadened lines and for field modulation frequencies $\omega_{m}$ such that $\omega_{m} \ll T_{1}^{-1}$. The $400 \mathrm{~Hz}$ modulation frequency used in the present experiment satisfied the latter condition when the carrier flow was sufficiently high, 


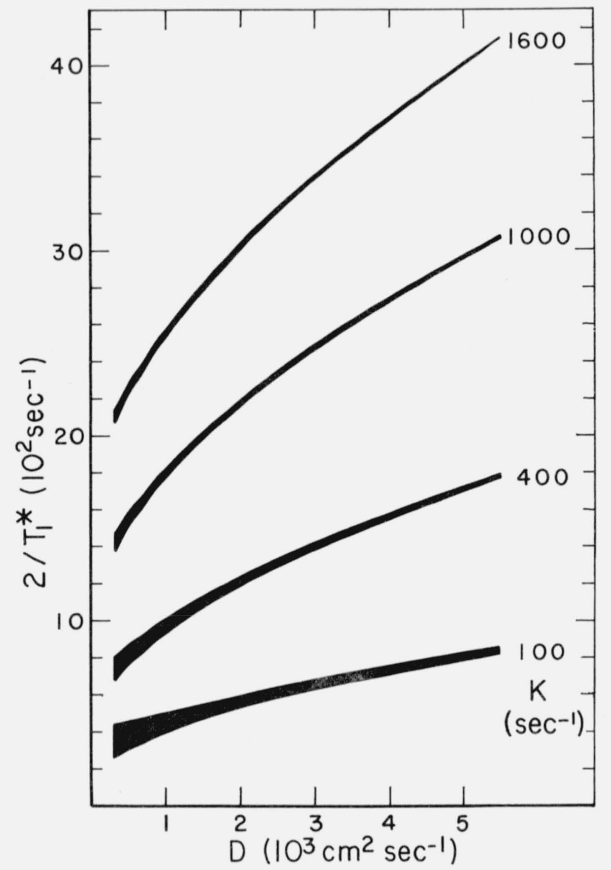

FIGURE 3. Variation of $2 / \mathrm{T}_{1}^{*}$ versus $\mathrm{D}$ calculated from eqs 11 and 12 for different values of $\mathrm{K}$.

For each $K$ value, $v_{0}$ was varied over the range 100 to $500 \mathrm{~cm} \mathrm{~s}^{-1}$; the effect of this on $2 / T_{1}^{*}$ is indicated by the width of each curve.

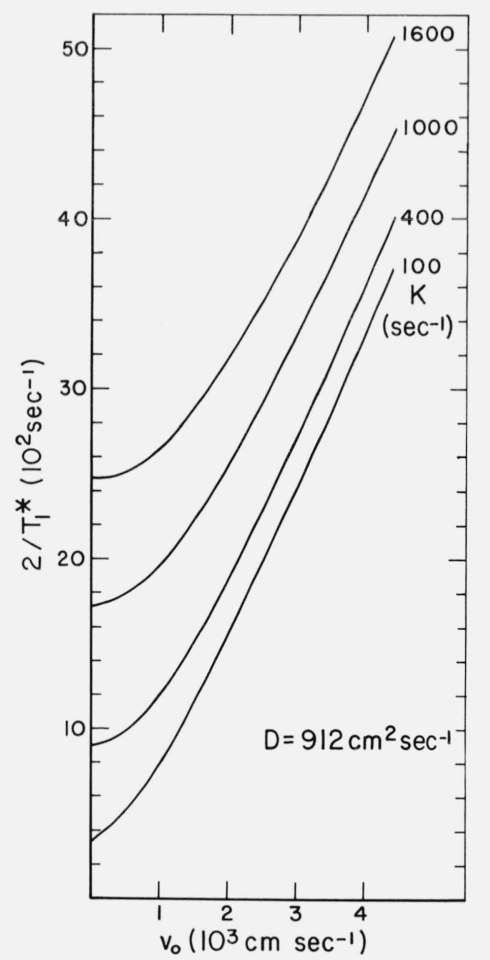

FigURE 4. Variation of $2 / \mathrm{T}_{1}^{*}$ with $\mathrm{v}_{0}$ at $\mathrm{D}=912 \mathrm{~cm}^{2} \mathrm{~s}^{-1}$, calculated from eqs 11 and 12 for different values of $\mathrm{K}$. but the former requirement was not satisfied since the lines were asymmetrical and presumably broadened by field inhomogeneities.

\subsection{Results of Saturation Experiment}

The signal enhancement produced by traces of $\mathrm{O}_{2}$ demonstrated that the $\mathrm{H}$ atom spin-lattice relaxation time in the present system was quite long. In this case, as can be seen from figure 4 , the relaxation time observed in a saturation experiment should depend markedly on the rate at which unsaturated atoms enter the cavity. The effect of carrier velocity on the amount of saturation produced by a given microwave power level is shown in figure 5 . Here, a $\mathrm{H}_{2}$ carrier was used at 2 torr with $v_{0}=1160$ and $3239 \mathrm{~cm} \mathrm{~s}^{-1}$. The high frequency $\mathrm{H}$ atom line is shown at a low power level at which saturation was negligible, and at a power level $20 \mathrm{~dB}$ higher for which considerable saturation was evident. In the double attenuator method used [1], the signal is proportional to $\chi^{\prime \prime}$, the imaginary part of the magnetic susceptibility. It is obvious that there was considerably less saturation at the higher power level for the higher carrier flow. In this experiment, about $40 \mathrm{ppm} \mathrm{O}_{2}$ was added to the carrier to ensure complete relaxation of the atoms arriving at the cavity so that the signal observed at low power would be proportional to the $\mathrm{H}$ atom concentration. This concentration was the same for both carrier velocities. The conditions of this experiment correspond to the third

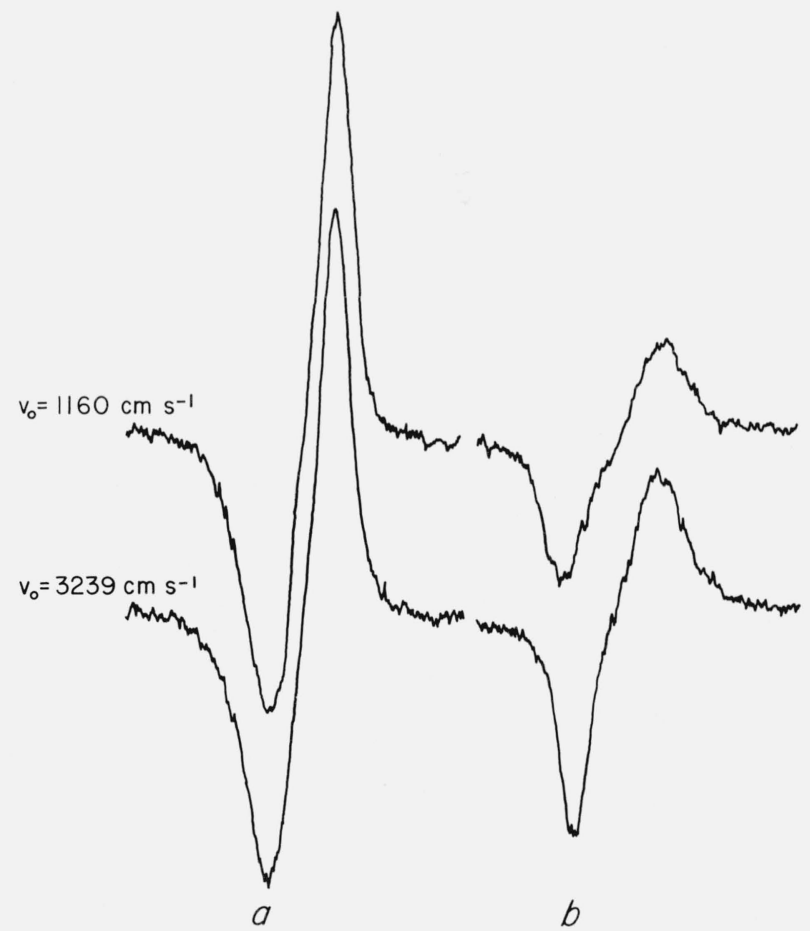

FIGURE 5. Effect of carrier flow velocity on the power saturation of the high-field $\mathrm{H}$ atom EPR line.

The two traces labeled (a) were recorded at low power where saturation was negligible. The $\mathrm{H}$ atom concentration is the same in both traces but the carrier velocity was different as shown. The traces (b) were recorded at a power level $20 \mathrm{~dB}$ higher. The lower trace in (b) corresponding to the higher carrier flow is less saturated than the one at the lower flow. 
curve in figure 4 , labeled $K=1000 \mathrm{~s}^{-1}$. This calculation predicts that a given power input would produce roughly half the saturation at $v_{0}=3239 \mathrm{~cm} \mathrm{~s}^{-1}$ that it would produce at $1160 \mathrm{~cm} \mathrm{~s}^{-1}$. The results shown in figure 5 are in agreement with this prediction.

\section{Discussion}

The purpose of this work has been to show how the $\mathrm{H}$ atom hyperfine populations equilibrate when the atom is placed in a magnetic field. In the present experiments there were two major relaxation processes, (a) spin-exchange collisions between the $\mathrm{H}$ atoms, and (b) spin-exchange collisions between the $\mathrm{H}$ atoms and $\mathrm{O}_{2}$ molecules added in trace amounts. The spin-exchange process conserves the component of angular momentum in the direction of the dc magnetic field, which we shall call the $z$-direction. Because states $\Psi_{2}$ and $\Psi_{4}$ have $z$ angular momentum components of zero, spinexchange collisions among the $\mathrm{H}$ atoms themselves can equilibrate [9] these levels without changing the net value of the $z$-component. The net magnetization of the system will change however, since these two states have nonzero magnetic moments at high field strengths. Thus, partial magnetization of the system will arise solely from $\mathrm{H}-\mathrm{H}$ collisions. It is worth noting that this situation cannot occur for $\mathrm{N}$ atoms, because none of the hyperfine levels have zero values for the $z$-component.

The other two states have nonzero components $+\hbar$ and $-\hbar$ for $\Psi_{1}$ and $\Psi_{3}$, respectively. Thus, the relative populations of these two levels cannot change without changing the total value of the $z$-component for the $\mathrm{H}$ atom system. This can be changed only through an interaction with the surrounding system, i.e., with the "lattice". Spin-exchange collisions with impurity $\mathrm{O}_{2}$ can provide the necessary spin-lattice interaction even though the $z$-component will be conserved during the collision. This is because any nonthermal spin orientation imparted to the $\mathrm{O}_{2}$ will be rapidly destroyed because of the strong coupling of the electron spin on $\mathrm{O}_{2}$ to its molecular framework. This is strongly coupled to the heat bath particles through collisions which change the $\mathrm{O}_{2}$ rotational energy.

From the enhancing effect of $\mathrm{O}_{2}$ on the $\mathrm{H}$ atom signal, a rate constant of $6 \times 10^{-10} \mathrm{~cm}^{3} \mathrm{~s}^{-1}$ was determined for the $\mathrm{H}-\mathrm{O}_{2}$ spin-exchange collision. Kleppner, Goldenberg, and Ramsey [14] examined the effect of $\mathrm{O}_{2}$ on the radiating state of the atoms in a hydrogen maser, and reported a relaxation rate constant. To compare our value with theirs, it is necessary to solve (7) for the zero-field case. Letting $\epsilon=\frac{1}{2}$ in tables 1 and 2 for $A_{i j}$ and $R_{i j}$, and neglecting diffusion and mass flow, one obtains for the population difference $\xi_{2}-\xi_{4}$ involved in the maser transition,

$$
\begin{aligned}
& \xi_{2}-\xi_{4}=E_{2}-E_{4}+\left(\xi_{2}^{0}-\xi_{4}^{0}-E_{2}+E_{4}\right) \exp \\
&(-4 \gamma t-K t)
\end{aligned}
$$

where $t$ is the time, and $\xi_{i}^{0}$ are the values of $\xi_{i}$ at $t=0$. If $4 \gamma \ll K$ in the presence of added $\mathrm{O}_{2}$, the relaxation will be represented by the change of $\exp (-K t)$ with time. Therefore the relaxation rate observed by these workers is equal to $K$ as defined in the present work. Their value of $K\left[\mathrm{O}_{2}\right]^{-1}=6.2 \times 10^{-10} \mathrm{~cm}^{3} \mathrm{~s}^{-1}$ is in agreement with ours. Neither of these values is in agreement with that of Westenberg and De Haas [5], whose $t_{1}$ values should be equal to $K^{-1}$ since their $\mathrm{H}$ atom concentration was kept high during $\mathrm{O}_{2}$ addition. (See eq. 10.)

A collision cross section $\sigma^{2}$ can be derived from the observed rate parameter $K \sim Q\left[\mathrm{O}_{2}\right]$ if one uses the kinetic-theory relation $K=\sigma^{2}(8 \pi k T / \mu)^{1 / 2}\left[\mathrm{O}_{2}\right]$, where $k$ is the gas constant, $T$ is the absolute temperature, and $\mu$ is the reduced mass of the colliding particles. The values of the rate constant $Q$ and the cross section $\sigma^{2}$ are shown in table 4 .

TABLE 4. Rate constant and collision cross section for $\mathrm{H}-\mathrm{O}_{2}$ spin-exchange collisions.

\begin{tabular}{l|l|c}
\hline \multicolumn{1}{c|}{$Q$} & \multicolumn{1}{c|}{$\sigma^{2}$} & Reference \\
\hline $6.4 \pm 2.0 \times 10^{-10} \mathrm{~cm}^{3} \mathrm{~s}^{-1}$ & $8.0 \pm 2.5 \times 10^{-16} \mathrm{~cm}^{2}$ & This work. \\
6.2 & 7.7 & {$[12]$} \\
35 & 44 & {$[5]$} \\
\hline
\end{tabular}

The cross section determined in the present work and by Kleppner, et al., is smaller than that for $\mathrm{H}-\mathrm{H}$ spin-exchange collisions. This may not be unreasonable since one would expect the attractive potential curve for $\mathrm{H}-\mathrm{O}_{2}$ to be considerably shallower than that for $\mathrm{H}-\mathrm{H}$ and thus the phase shift between the attractive and repulsive potentials could be smaller. However, since it appears that the steepness of the repulsive state potential curve is also an important factor, at least in the $\mathrm{H}-\mathrm{H}$ case [15], it is difficult to say anything definite about the $\mathrm{H}-\mathrm{O}_{2}$ system without a detailed knowledge of the states involved.

It is not clear why wall relaxation should have been so much more important in the experiments of Westenberg and De Haas than in ours. Nevertheless, our observations show that it is quite possible for wall relaxation to be negligible in some systems. In this situation some $\mathrm{O}_{2}$ impurity must be present to achieve complete magnetization. The amount necessary at moderate carrier flows is very small however, of the order 15 to $30 \mathrm{ppm}$, so that it should not interfere with kinetic studies of moderately fast reactions. Under such conditions however, power saturation can still depend on the carrier flow rate. A line which is unsaturated at a high flow rate may be saturated at a lower one.

\section{References}

[1] Brown, R. L., and Brennen, W., J. Chem. Phys. 47, 4697 (1967).

[2] McNeal, R. J., Bernheim, R. A., Bersohn, R., and Dorfman, M., J. Chem. Phys. 40, 1678 (1964).

[3] Brown, R. L., and Brennen, W., J. Chem. Phys. 46, 385 (1967).

[4] Breen, J. E., and Glass, G. P., J. Chem. Phys. 52, 1082 (1970).

[5] Westenberg, A. A., and De Haas, N., J. Chem. Phys. 5 1, 5215 (1969).

[6] Tollefson, E. L., and Leroy, D. J., J. Chem. Phys. 16, 1057 (1948). 
[7] Rony, R. P., and Hanson, D. N., J. Chem. Phys. 44, 2536 (1966)

[8] Breit, G., and Rabi, I. I., Phys. Rev. 38, 2082 (1931).

[9] Purcell, E. M., and Field, G. B., Astrophys. J. 124, 542 (1956).

[10] Lloyd, J. P., and Pake, G. E., Phys. Rev. 94, 579 (1954).

[11] Lambe, E. B., Thesis, Princeton University, 1959 (unpublished).

[12] Westenberg, A. A., and De Haas, N., J. Chem. Phys. 47, 1393 (1967).
[13] Clyne, M. A. A., and Thrush, B. A., Trans. Faraday Soc. 57, 1305 (1961).

[14] Kleppner, D., Goldenberg, H. M., and Ramsey, N. F., Phys. Rev. 126, 603 (1962).

[15] Glassgold, A. E., and Lebedeff, S. A., Ann. Phys. 28, 181 (1964). (Paper 76A2-703) 